\title{
GÉOGRAPHIE ET NUDITÉ: UN MARIAGE EXTRAVAGANT? (QUELQUES PISTES DE RÉFLEXION SUR LA PRODUCTION SOCIALE DE L'ESPACE)
}

\author{
Francine Barthe-Deloizy*
}

\section{RÉSUMÉ:}

Est-il possible d'aborder la nudité par l'angle improbable de la géographie ? La géographie auraitelle quelque chose à dire de plus, de différent sur le sujet? De prime abord la question semble incongrue. Après tout quel peut bien être le rapport entre géographie et nudité ? Hormis le fait de produire un mariage étrange, une association insolite Ce travail propose de montrer que l'entrée par les lieux et les territoires permet de mieux comprendre le (les) sens de la nudité, et montre par la même occasion comment le corps, plus ou moins dévoilé est impliqué dans la production sociale de l'espace. Cet article se présente donc comme une invitation à visiter ces espaces de la nudité et à réfléchir sur les corps qu'on y voit.

\section{MOTS-CLEFS:}

Nudité; espace; géographie; corps.

\section{ABSTRACT:}

Is it possible to approach nudity by the improbable standpoint of geography? Would geography have anything to add on the subject, anything different to tell us? To begin with, the question seems inappropriate. After all, what can be the connection between geography and nudity? Except the fact of producing a strange combination, an unusual association. This paper is intended to demonstrate that entering places and territories makes it possible to better understand the meaning(s) of nudity, and consequently shows how the body, more or less revealed, is involved in the social production of space. Thus this article is conceived as an invitation to visit these spaces of nudity and consider the bodies one might see in them.

\section{KEY WORDS:}

Nudity; space; geography; body. \section{insolite}

Géographie et nudité : un mariage

Est-il possible d'aborder la nudité par I'angle improbable de la géographie ? La géographie aurait-elle quelque chose à dire de plus, de différent sur le sujet? De prime abord la question semble incongrue. Après tout quel peut bien être le rapport entre géographie et nudité ? Hormis le fait de produire un mariage étrange, une association insolite! Nous relevons ici ce défi qui fait écho à une publication $^{1}$. Ce travail propose de montrer que
I'entrée par les lieux et les territoires permet de mieux comprendre le (les) sens de la nudité, et montre par la même occasion comment le corps, plus ou moins dévoilé est impliqué dans la production sociale de l'espace. Cet article se présente donc comme une invitation à visiter ces espaces de la nudité et à réfléchir sur les corps qu'on y voit. Le fil conducteur de ce travail n'est donc pas l'étude de la nudité en soi, objet épistémologique que nous empruntons aux anthropologues, aux historiens ou aux sociologues... mais des espaces que la nudité 
Géographie et nudité: un mariage extravagant? (quelques

pistes de refléxion sur la production sociale de l'espace), pp. 152 - 169

produit. La nudité individuelle ou collective, privée ou publique, crée des lieux, des territoires, des pratiques, travaillées par des normes, des codes, des histoires, des morales et des idéologies. C'est dans la confluence de ces productions que se situe l'objet de cette étude.

A y regarder de plus près l'enjeu de cet essai réside dans le regard qu'on porte sur la nudité, regard qui suit des perspectives différentes selon l'endroit, et l'époque où I'on se trouve. L'espace, du fait des corps nus se métamorphose et les pratiques qui s'y inscrivent également. La nudité s'inscrit ainsi dans une géographie culturelle qui fait qu'elle n'est pas la même en Allemagne, en France, au Brésil ou en Iran. Que la nudité de I'homme blanc n'est pas celle de la femme noire.. surtout aux yeux du premier!

Le formidable pouvoir de fascination qu'exerce le corps nu sur les regards est bien une caractéristique fondamentale de ce sujet et elle n'a rien de contemporain comme pourrait le laisser croire les Unes de couvertures des journaux (masculins ou féminins, de charme, de bien-être et santé ou tout simplement de la presse grand-public), en effet la chose n'est pas nouvelle puisque, on retrouvait déjà dans les journaux de voyage des grands découvreurs du XVe siècle cette même stupéfaction ${ }^{2}$. Devenu un sujet de société, quoique le dénuder qu'il convient de ne pas confondre avec nudité, soit plus attractif aux dires des professionnels des médias.

\section{pratique \\ Archéologie d'un mot, généalogie d'une}

Commençons par une évidence : la nudité en soi ne signifie rien, ne dit rien et se réduit finalement à un simple état, celui d'un corps que ne recouvre aucune enveloppe, aucun signe.

Cette incroyable neutralité s'efface dès lors qu'on la met en perspective avec un lieu, un contexte, en d'autres termes lorsqu'on la met en situation. Se mettre nu sous sa douche relève d'une grande banalité, mais se rendre à l'opéra ou au restaurant intégralement nu est considéré comme de l'exhibitionnisme ou de la provocation. Ces exemples caricaturaux montrent d'emblée qu'une distinction opératoire se dessine. Dans le premier cas, il est question d'une pratique corporelle hygiénique qui se déroule au sein de l'espace domestique, dans l'autre d'une pratique culturelle impliquant des règles de sociabilités convenues dans I'espace public. La nudité se résumerait-elle à ce double binôme individu/collectif-privé/public dans le cadre de pratiques sociales? Une question se pose alors : quel statut accorder à la nudité, de la tolérance à son égard ? La nudité serait-elle une sorte de caméléon culturel qui ne se comprend que par rapport à un contexte, une époque ? La nudité est mise en jeu dans toutes les interactions avec autrui, le travail, le sport, la santé, mais aussi dans les pratiques les plus intimes, comme par exemple I'hygiène ou la sexualité.

L'étymologie latine de nudité (nudus) nous apprend que le terme ne signifie pas nécessairement absence totale de vêtement. Pour les romains, être nu, c'est ne pas se couvrir le corps comme l'exigent la situation et le lieu où I'on se trouve sous peine d'être choquant ou inconvenant. On retrouve cette complexité dans l'acception proposée par le dictionnaire de la langue française qui définit la nudité par rapport au vêtement « qui n'est couvert d'aucun vêtement : être dans le costume d'Adam et Eve, dans le plus simple appareil, dans l'état de nature... » D'autres approches proposent une dichotomie plus nette insistant sur le caractère sexué. Pour M.A Descamps ${ }^{3}$ est nue «toute personne dont les organes sexuels ne sont pas masqués par un objet adéquat, et vêtue celle qui cache au moins une partie de son corps ». L'amalgame établi entre sexe et nudité représente la caractéristique majeure de la civilisation judéo-chrétienne, là plus qu'ailleurs la nudité s'est résumée pendant des siècles au dévoilement du sexe.

Au fil de l'histoire, et sous des prétextes variés, la nudité a été parée de valeurs, de normes, de tabous. Elle est tour à tour, scandaleuse, excitante, morbide ou innocente. Elle constitue un vocabulaire, un langage qui aide à comprendre les sociétés dans lesquelles elle se donne à voir ou plus 
exactement se laisse deviner. Derrière l'énonciation et la prééminence d'une manière de voir le nu, se manifestent des enjeux de savoir et de pouvoir ; c'est la raison pour laquelle les religions comme les idéologies ont paré le nu de toutes les vertus et de tous les vices. Ainsi, dans l'aire judéo-chrétienne, dès l'antiquité, le corps fit l'objet de prescriptions contradictoires et la nudité innocente, celle du paradis d'Adam et Êve a côtoyé pendant des siècles la nudité pécheresse ou infamante des damnés.

\section{Les signifiés de la nudité}

Les hommes ne se sont jamais mis par hasard, par caprice ou par envie, n'importe où ni dans n'importe quelles circonstances. Si I'homme naît nu, à l'évidence la société I'habille, le pare, le décore...et pourtant, I'histoire de I'humanité nous montre des êtres qui passent leurs temps à se déshabiller plus ou moins discrètement, ostensiblement à certaines périodes, en certaines occasions. C'est dans cette activité de va et vient continuel que surgit le phénomène de nudité : c'est à dire précisément l'acte de rendre au corps une partie ou l'intégralité de son état originel. La nudité n'a jamais été vécue comme un fait brut et insignifiant, c'est la raison pour laquelle il convient de ne pas confondre la nudité et le dénuder, deux phénomènes majeurs et pas nécessairement concomitants se sont produits : le fait et la conscience de se dévêtir. Ce constat initial invite à s'interroger sur les lieux précis qui font et permettent qu'un corps habillé, paré, masqué retrouve sa nudité première.

Les usages de la nudité se résument à des attitudes précises, mettant en jeu un binôme bienêtre/mal être qui se définit suivant les époques ou les aires culturelles au travers de pratiques qui impliquent et parfois remettent en cause les normes de pudeur. Dans l'expérience culturelle des peuples et plus précisément dans la tradition occidentale, le dénuder paraît investi de trois significations principales : l'expression d'un bien-être, le partage d'une intimité avec un entourage et le symbole d'une humiliation car le dénuder peut aussi devenir un signe d'infamie.

A chaque aire culturelle correspondrait donc une définition de la nudité et de ses valeurs, des gestuelles, des pratiques, des lieux spécifiques. L'horizon de départ de cette recherche a été la culture judéo-chrétienne. On pourra sans doute reprocher l'aspect européano-centré de ce travail, mais il faut bien admettre qu'une grande partie du monde par le biais de la christianisation, puis de la colonisation a imprimé une trace indélébile sur la nudité de ceux qu'on appelait il y a peu de temps les peuples primitifs.

\section{La mise aux normes de la nudité des « sauvages 》}

« Nus donc sauvages, habillés donc civilisés » cette formule lapidaire servit longtemps de norme pour définir un « niveau de civilisation ». A l'évidence, la nudité (mais pas seulement) a lourdement contribué à ce classement entre peuples « archaïques » et « peuples évolués », ce qui permet au passage de mettre en évidence une forme ethnocentrique de définition de ce qui est ou pas une civilisation. Très tôt une analogie entre l'état de maturité ou d'immaturité des cultures a été établie en fonction de ce critère. Quelque soit la terminologie choisie : sauvages, primitifs, archaïques ces peuples ont été vus et observés en tant que peuples nus et c'est par le regard porté sur cette nudité qualifiée de naturelle et naïve (dans sa version rousseauiste) ou immorale (version religieuse) qu'ils ont été caractérisés.

La relative simplicité des critères établis dans le contexte positiviste du XIXe siècle a permis de mettre en place cette discrimination, souvent associée d'ailleurs à un état proche de la barbarie ou de la sauvagerie. Les théories darwiniennes définissaient l'indigène comme un primitif sorti tout droit de la préhistoire et dont l'évolution s'était arrêtée à l'âge de pierre. La nature c'était le sauvage échappant par définition à la civilisation. En vivant dans le plus simple appareil, ils appartenaient à la nature au même titre que les plantes ou les animaux. Comme la nature, les indigènes étaient menaçants jusqu'à ce qu'on parvienne à les contrôler. Le devoir 
Géographie et nudité: un mariage extravagant? (quelques

pistes de refléxion sur la production sociale de l'espace), pp. 152 - 169

de « I’homme blanc » était donc d'enseigner aux indigènes ignorants et puérils comment vivre et travailler de façon productive donc civilisée. Les grands principes de la civilisation d'édifièrent sur deux idées apparemment contradictoires, il fallait d'une part faire cesser des pratiques barbares comme l'anthropophagie, mais en même temps il convenait de préserver cette supposée conception indigène de la nature.

C'est dans la droite ligne de cette idéologie romantique du «sauvage » que s'organisèrent des zoos humains dans toute l'Europe dans le cadre d'exposition coloniale (de la fin du XIXe siècle jusque dans les années 1930$)^{4}$. La nudité emblème de l'indigène noir devint donc pendant toute la période coloniale signe de sauvagerie, il convenait de la faire disparaître en habillant les sauvages. Ce qui fut fait, mais sous des couverts moralisateurs « vêtir ceux qui sont nus » ce zèle évangélisateur permis d'ouvrir un marché du textile de 320 millions de cotonnades anglaises. Une aubaine commerciale! ${ }^{5}$

Ainsi au nom de cette différence marquée entre peuples conquérants et peuples conquis se sont mis en place des systèmes d'évaluation qui indiquait que l'Autre, parce qu'il était nu, était inférieur et à ce titre dévalué et déprécié. En habillant les sauvages, les sociétés coloniales ont témoigné de leur volonté de retrouver à toute force le même sous l'Autre en niant leur Altérité.

\section{La nudité comme marqueur spatial:}

La nudité passée la naissance, se définit comme un acte, un geste. Nous revenons ici sur notre hypothèse de départ: le lieu sert de modalités d'évaluation de la nudité. Être nu sur une plage appartient (au moins dans la plupart des pays d'Europe) au paysage balnéaire courant depuis plusieurs décennies maintenant, en revanche que des hommes ou des femmes manifestent nus pour revendiquer (le droit à leurs terres, des pistes cyclables ou leur hostilité à l'égard des vêtement en fourrure) I'est beaucoup moins. Que penser aussi des pitreries des streakers nus, extravagance des pays anglosaxons qui surgissent au milieu de la foule dans les grandes rencontres sportives internationales de football de rugby ou tennis? Le lieu sert dans toutes ces situations de marqueur spatial à la nudité.

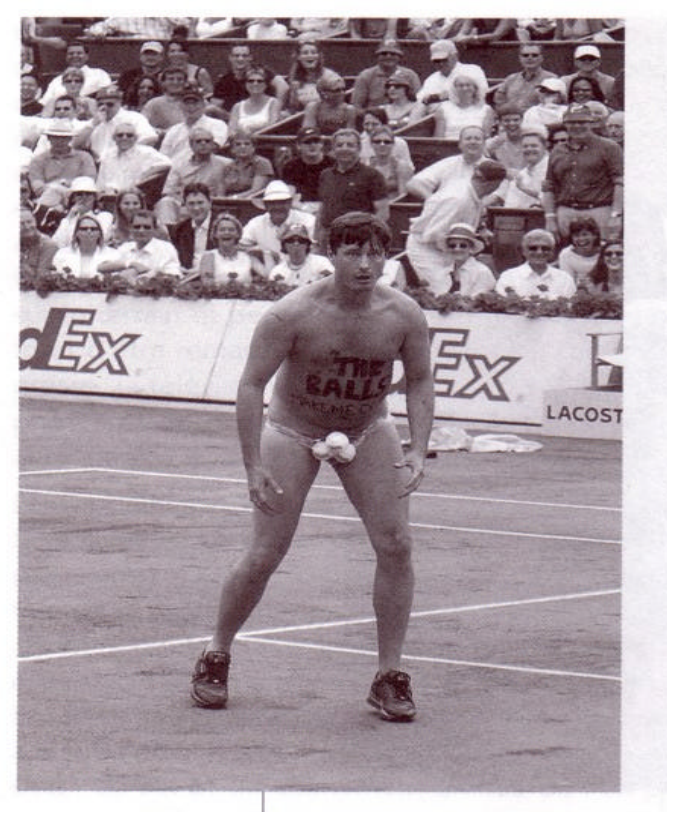


Cette situation du nu ordinaire ou exceptionnel, spectaculaire ou banal a pour effet de caractériser les lieux dans lesquels la nudité a sa place ou ne l'a pas. La nudité produit des lieux réservés à l'hygiène, collectifs ou individuels, mixtes ou non mixtes: depuis des siècles, les fonctions corporelles qui nécessitent la nudité ont déterminé la création d'édifices comme les bains douches, les hammams dans les pays d'orient ou d'espaces particuliers dans les maisons (la salle de bain par exemple). L'intimité signalée par la nudité indique le passage à la sphère du privé. Le vêtement insigne des statuts publics et des codes sociaux (rappelons que le vêtement a trois fonctions : la protection, l'ornementation et la communication) perd une large part de ses attributs lorsque l'individu quitte le groupe pour rentrer chez lui.

Des environnements particuliers se sont ainsi développés pour permettre à la nudité et à un entre-soi d'exister. Ces espaces s'intègrent à l'organisation de la vie quotidienne et deviennent ainsi partie prenante des rythmes de la vie en produisant des temps particuliers dédiés à la nudité. Ce qui est symptomatique c'est que quelque soit le lieu considéré il répond à un système de normes liés à des contextes, à des temporalités, à des cultures, à une gestuelle organisée pour bien signifier que la nudité corporelle n'a rien d'innocent ni de naturel et qu'elle doit s'entourer d'une panoplie très compliquée de précautions, avec des règles précises qui ne sont pas toujours formulées par des mots, des ordonnances religieuses ou des lois. De ce point de vue la nudité relève bien d'une construction culturelle. Les contextes commandent donc des gestuelles particulières, surtout lorsqu'il y a mixité dans un même lieu. Ces stratégies corporelles démontrent l'existence d'une correspondance globale entre l'utilisation que les individus font de leur corps et la culture du groupe auquel ils appartiennent, groupe social mais aussi groupe sexué.

\section{Les lieux pour l'intimité, l'hygiène et les soins du corps}

Les pratiques corporelles nécessitant la nudité diffèrent suivant les aires culturelles ou les époques mais dans tous les cas, elles créent des lieux spécifiques. Hammam, sauna, salles de bain s'inscrivent dans l'espace urbain comme dans l'espace domestique et sont là pour attester que la nudité produit et organise l'espace.

- Hammam et sauna : nudité et lieux de sociabilité

Dans les pays du Maghreb ou d'Orient, le hammam marque de sa présence toutes les villes. Il appartient aux cérémonies de I'hygiène corporelle et aux rituels de purification. Ils ont influencé les règles de vie des musulmans. Ces bains sont I 'occasion de soins précis: lavage du corps, friction, lavage des cheveux et épilation. Le Hammam est un bain à étuves, il s'agit d'un édifice typique du monde islamique autour duquel gravite la vie d'un quartier, il est au centre de la vie des croyants et en même temps un centre de rencontres et de vie sociale. Pour Malek Chebel ${ }^{1}$ avec la mosquée le hammam est un fil tendu par dessus la ville islamique. Malgré la présence aujourd'hui dans les maisons citadines des éléments de confort, le hammam reste un haut lieu de convivialité. Dans le hammam il n'existe aucune confrontation directe entre les hommes et les femmes, il est tout à la fois un salon de coiffure, un dispensaire de kinésithérapie (massages), une agence matrimoniale (c'est là qu'on arrange les mariages), un lieu de rendez-vous.

Dans les pays du Nord de I'Europe, le bain de chaleur sèche (le Sauna) est le marqueur de civilisation des pays scandinaves. Loin d'être une mode, il est de tradition très ancienne. A l'origine il est lié à I'héritage des traditions rurales, le sauna est un bain familial compris au sens large 
Géographie et nudité: un mariage extravagant? (quelques

pistes de refléxion sur la production sociale de l'espace), pp. 152 - 169

du terme, la maisonnée ou la famille élargie. Dans ce lieu, qui à l'origine était une petite cabane en bois établie à proximité de la ferme il est tout à la fois l'endroit ou les femmes allaient accoucher, le lieu ou on se retrouve entre voisins ou avec la famille élargie. C'est aussi la pharmacie du pauvre puisqu'on y pratique les petites opérations, les vielles personnes viennent $s^{\prime} y$ réchauffer...et toute générations confondues on y pratique une nudité conviviale mixte et intergénérationnelle. Il fait son apparition aux Etats Unis par le biais de familles de migrants dès le XIXe siècle, puis devient un phénomène de mode à partir des années 1960 par l'intermédiaire des athlètes américains qui l'utilisent pour les jeux olympiques. Aujourd'hui le sauna appartient à la panoplie du confort et du mode de vie contemporain.

- Des bains publics à la salle de bain : nudité et hygiénisme

Les prescriptions de propreté mises en place par les hygiènistes, la nécessité du lavage, de la toilette sont à l'origine de l'édification et de l'organisation d'établissements de bains publics sanitaires, les bains douches collectifs. Ce qui semble évident aujourd'hui mérite cependant quelques précisions. La nudité et I'hygiénisme ne vont pas de soi, en effet jusqu'au XVIIIe siècle, I'eau était considérée comme suspecte et vecteur de tous les maux (les miasmes). La toilette se réduisait alors aux endroits les plus visibles du corps: les mains, le visage et la bouche. A cette méfiance de l'eau sur le plan médical s'ajoutait aussi la défiance à l'égard du corps sur le plan religieux. La nudité s'avérait dangereuse pour les puritains. Il faut attendre le traité d'hygiène de Lavoisier en 1777 puis les idées du retour à la nature préconisée par les livres de JJ Rousseau pour voir apparaître les premiers principes $d$ 'hygiène de vie (le mot est nouveau). Ces idées d'avant garde ne touchent à l'époque que les élites urbaines. Ce changement de statut, se manifeste par un renouvellement des pratiques : le bain devient bain de propreté et le savon se transforme en outil de santé. Mais les normes de pudeur auront encore la vie dure et elles constituent un frein au progrès de l'hygiénisme. Les prescriptions de propreté mises en place par les hygiénistes, la nécessité du lavage, de la toilette sont à l'origine de l'édification et de l'organisation d'établissements publics sanitaires, les bains douches collectifs. Aujourd'hui quasiment tous disparus du paysage urbain, ils laissent la place à la salle de bain.

Ce rapide panorama (qui n'a qui n'a aucune prétention à l'exhaustivité) des lieux d'hygiène et de soins du corps, contribue à expliquer et à enrichir la notion d'intimité. La relation entre intimité et nudité traduit un rapport à soi et aux autres en instaurant des écarts, des distances entre les individus. Selon les cultures et les contextes, chaque individu appartenant à un groupe ou à un genre adopte des stratégies particulières en fonctions des situations dans lesquelles il se trouve. Nous reprenons à cet égard les travaux de E T Hall ${ }^{2}$ qui parle de distance intime en mode proche ou éloigné. Cette distance particulière est celle ou la présence de l'autre s'impose et peut même devenir envahissante. Ainsi dans les bains publics, dans les sauna ou les hammams ou dans les bains japonais (les Onsens) là ou se côtoient des groupes monosexués (hammam) ou mixtes (sauna ou Onsens japonais), la nudité n'est ni mixte ni innocente. La co-présence d'individus des deux sexes nécessite et produit ce que Hall nomme des structures proxémiques. Les regards évitent certaines parties du corps du voisin ou de la voisine. $C^{\prime}$ est ce que HP Duerr ${ }^{3}$ nomme « un mur invisible ou un regard fantôme ».

Avec I'avènement de I'habitation privée, et la diminution relative des pratiques collectives d'hygiène et de soins 
apportés au corps on peut dire aujourd'hui que la nudité est devenue un marqueur discriminant de l'espace de vie. Désormais il y a des lieux au sein de l'espace domestique ou le corps peut se dénuder, se déployer librement et les lieux ou il n'est présenté que dans la mise en scène. La salle de bain, parfois la chambre à coucher dans tous les intérieurs sont bien les endroits d'où l'on peut s'exclure des autres, du public. Dans ces lieux réservés à la nudité s'instaure un dialogue de familiarité et d'intimité. C'est la raison pour laquelle lorsque la le corps nu fait irruption dans l'espace public en sortant de cette sphère de l'intimité, il transgresse un code et devient incongru, totalement insolite.

\section{public ?}

Le nu a-t-il sa place dans l'espace

La nudité appartient à la sphère du privé et à l'intimité, si elle s'affiche à l'extérieur, dans l'espace public elle sort de son statut traditionnel et provoque une perturbation de la notion même d'espace public. Différents types de nudité concernent l'espace public: le nu balnéaire, ou nudisme, le nu protestataire, le nu festif (nudité affichée lors d'évènements urbains de type love parade ou Gay sont des expressions collectives ou individuelles appartenant au langage déployé par le corps nu dans l'espace public. Dès lors, quelle est la fonction de l'espace public au regard de ces différents types de nu. Nous postulons ici que le nu est susceptible de renforcer ou de minorer le caractère public de l'espace (porteur de civilité, d'anonymat, domaine du collectif, de l'extimité ${ }^{4}$ ) jusqu'à parfois le modifier en son contraire, c'est à dire en espace intimiste ou communautaire. Tout se rapporte alors à la question du nu, du regard qu'on porte sur lui et de la valeur qu'on attribue à l'espace public. Dans certaines situations, la nudité est considérée comme exhibitionniste, alors que dans d'autres elle est largement tolérée, pourquoi ?
- Le nu et la loi

La nudité n'a jamais fait bon ménage avec l'espace public, en témoignent les nombreuses lois qui l'ont sanctionné partout dans le monde. Signalons toutefois que la permissivité, la tolérance à son égard est un marqueur de démocratie. En France le code pénal (article 222-32) de 1994 pénalise l'exhibition sexuelle «imposée à la vue d'autrui dans un lieu accessible à la vue du public » (peines encourues 15000 euros d'amende et un an d'emprisonnement).

Une grande disparité entoure la perception de la nudité, la plupart du temps sa définition relève de l'arbitraire. Pour les sociétés habillées la nudité se confond avec une représentation de la sexualité humaine elle se réduit à des organes corporels. C'est pourquoi, si l'on cherche à définir la nudité uniquement par la mise à jour des organes sexuels il convient aussi de préciser quels sont les organes que I'on considère comme sexuels pourquoi on en retient certains et pas d'autres et jusqu'ou on peut aller dans la mise à jour. La conscience (la définition) de la nudité se construit à partir d'un objet à la fois étrange et familier, producteur d'identités mais aussi de leurres: le corps. Chaque espace, chaque période, chaque société développe sa propre nudité à ce titre celle-ci obéit à des règles à des rituels à des mises en scènes quotidiennes et même à des débordements. Bien que l'ensemble soit codifié, son observation et son analyse demeurent toujours problématiques.

\section{- Le nu balnéaire}

La plage plus que tout autre territoire, raconte l'épopée de la dénudation. L'observation d'une plage de Rio ou des rivages iraniens de la mer Caspienne sont éloquents pour illustrer cette comparaison. Ici, le déploiement de corps musclés, retouchés par la chirurgie esthétique, huilés, 
Géographie et nudité: un mariage extravagant? (quelques

pistes de refléxion sur la production sociale de l'espace), pp. 152 - 169

bronzés indiquent un culte de la beauté, d'une sexualité libre et de la jeunesse quasiment éternelle, là bas: des plages séparés par des rideaux ou d'un côté les dames se baignent entre elles en tenue islamique, de l'autres les hommes en maillot de bain et sans gestes équivoques des un(e)s à l'égard des autres. Une autre comparaison peut s'établir aussi entre les plages du nord au sud de l'Europe et les plages brésiliennes. Ici, se déploie depuis les années 70 une mode des seins nus de I'autre côté de l'Atlantique malgré la surface réduite à son minimum des maillots de bains (I'Europe doit au Brésil l'usage du string ou fil dentaire) la pratique du topless sur les plages brésiliennes est rarissime. On l'aura compris ces quelques exemples n'ont qu'une valeur d'exemple détournée car de nudité il n'est pas question ici mais plus précisément de « dénuder».

\section{- Nudisme et naturisme}

Le processus de dénudation des corps s'est effectué de manière lente mais inéluctable dans toute l'Europe, jusqu'à aboutir à une dénudation complète qui tend à devenir un phénomène banal sur de nombreuses plages d'Europe et particulièrement en France. La pratique de la nudité intégrale sur les plages ou dans des espaces de nature apparaît (les forêts, les lacs et parfois les parcs publics comme c'est le cas en Allemagne) comme une originalité et une spécificité européenne. Elle a donné lieu à deux types de pratiques de la nudité qui ne doivent pas être confondues. Le nudisme, essentiellement localisée sur les plages et pendant la période des vacances est une pratique occasionnelle, et sans doute le signe d'une longue évolution des normes de pudeur. Son objectif est l'exposition intégrale du corps au soleil pour le bronzage. Le naturisme est une idéologie de la nature qui naît en Allemagne au XIXe siècle ${ }^{5}$, il prend sa source au carrefour des philosophies de la nature, d'une médecine néo-hippocratique qui utilise les éléments de la nature à des fins thérapeutiques en pratiquant des cures naturistes (bain de mer, de lumière, de pluie), enfin il s'inscrit dans une contestation de l'ordre dans le cadres des mouvements de réforme de vie de la jeunesse allemande (lutte contre la civilisation industrielle et ses méfaits). En France C'est à partir des années 20 que le naturisme acquiert une certaine popularité auprès des élites urbaines. La pratique de la nudité intégrale, mixte et familiale soulève une vague de protestations et nombreux seront les procès intentés contre les pionniers du naturisme. Les lois d'outrage public à la pudeur, largement hostiles au mouvement expliquent I'enfermement et l'organisation du naturisme dans des centres longtemps appelés camps de naturistes par les non pratiquants. Depuis les années 50 le naturisme s'est doté de structures touristiques ${ }^{6}$ (les centres de vacances naturistes, les plages naturistes) qui sont gérés et contrôlés par un organisme fédérateur (la FFN). La France est devenue la première nation naturiste du monde en terme de capacité d'accueil, elle reçoit chaque années environ 2 millions de naturistes dont la moitié sont des étrangers venant du nord de l'Europe.

Ainsi, sur les plages se côtoie un public de nudistes/naturistes pratiquant la nudité intégrale, mais un problème demeure : celui du rapport à la loi ! Les plages appartiennent au domaine public de I'Etat et les maires des communes du littoral doivent y faire appliquer la loi. Deux situations se dessinent : certaines communes autorisent sous la forme d'arrêté municipal la pratique de la nudité intégrale sur les plages sur un secteur limité et balisé par une signalétique, la plupart du temps ces arrêtés entérinent une pratique déjà établie depuis longtemps. Partout ailleurs, la nudité est interdite et tombe sous le coup de la loi d'outrage public à la pudeur. Malgré ces interdictions la pratique de la nudité se 
réalise sur les marges, loin des accès faciles, les plages nudistes sont cachées et connues par un public qui se transmet de bouche à oreilles l'emplacement des sites. Se dessine ainsi, une géographie des lieux naturistes et nudistes en France.

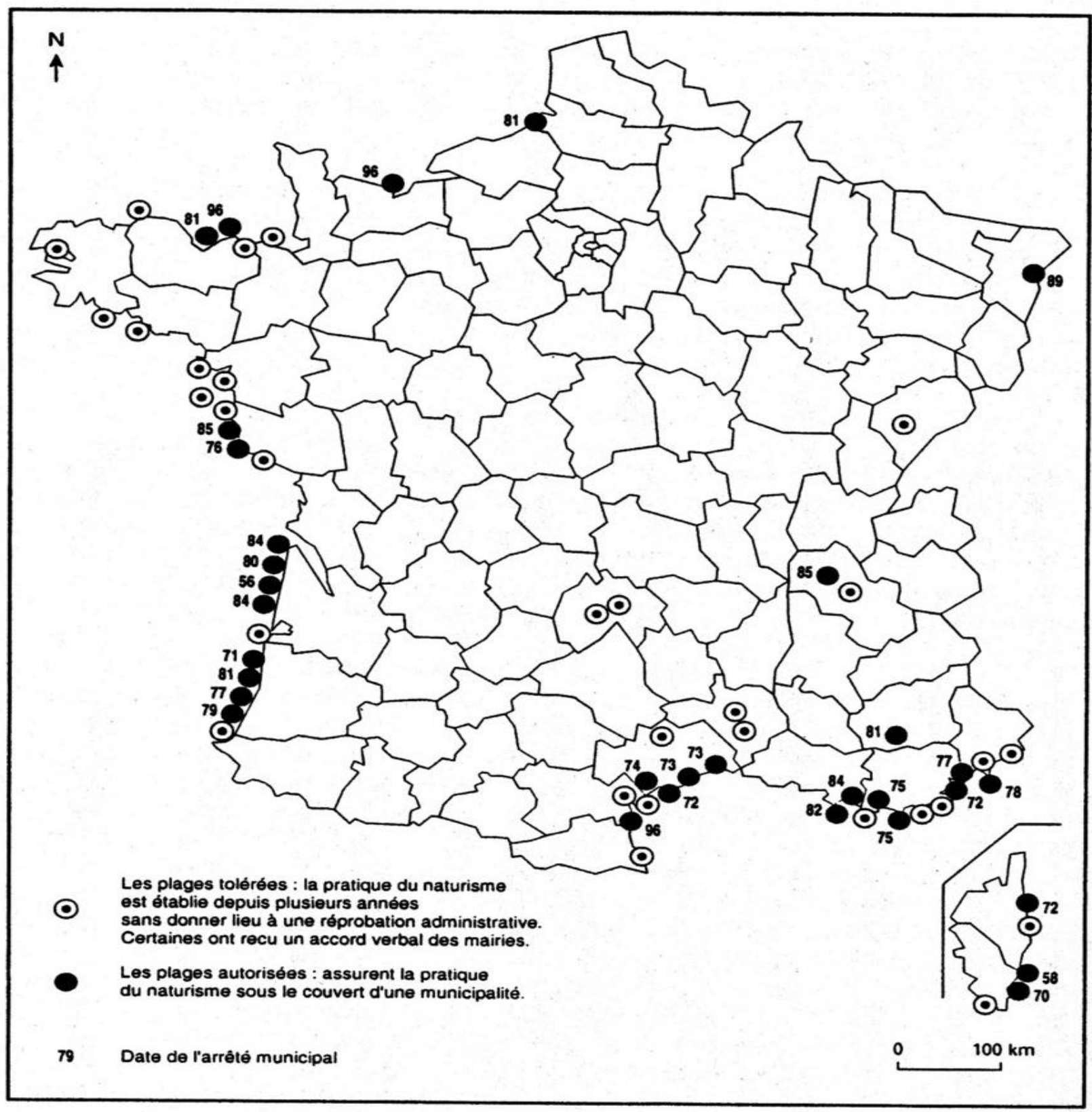


Géographie et nudité: un mariage extravagant? (quelques

pistes de refléxion sur la production sociale de l'espace), pp. 152 - 169

La dénudation du corps sur les plages produit un découpage de l'Europe, un signe de différenciation culturelle supplémentaire. Dans l'Europe du sud, la tolérance est faible, alors que dans toute I'Europe du Nord la nudité sur les plages est banale et non réprimée. L'impact de la religion a sans aucun doute une influence sur ce découpage.

\section{De la fête à la contestation de I'ordre social : le nu « porte parole»}

L'acte de dénudation se veut aussi contestataire de l'ordre social. Ces manifestations nues adoptent plusieurs formes: elles peuvent être festives, religieuses ou s'inscrire dans un registre plus large de contestation idéologique. La chose n'est pas nouvelle, elle est attestée dès l'antiquité lors des saturnales romaines, plus tard au moyen âge, lors des fêtes des innocents ou pour l'arrivée du roi dans une ville on organisait des mystères spectacles nus vivants racontant des scènes mythologiques. Mais c'est surtout au cours du XXe siècle et plus particulièrement dans les années 70 que la dénudation provocante s'est manifestée avec le plus d'intensité. D'abord dans les milieux anti-conformistes artistiques de la contre-culture du monde anglo-saxon puis dans les cercles hippies. La dénudation individuelle a ensuite pris un aspect résolument spectaculaire avec le phénomène du streaking (de l'anglais to streak qui signifie filer comme l'éclair) qui pourrait se résumer à une manière d'amuser la galerie en faisant le clown intégralement nu et si possible lors d'une manifestation officielle.

Avatar sans doute de ces fêtes joyeuses et débridées médiévales, le carnaval, les gay pride, love parade ou techno parade sont aussi des occasions de voir défiler dans les rues des fêtards dans des états de nudité plus ou moins abouti. De Rio à Paris, de Berlin à New York ces parades ne sont pas à proprement parlé des lieux où se donnent à voir des spectacles de nus, en revanche le déshabillé y est largement évoqué, La nudité intégrale y côtoie parfois un nu à peine voilé dont la connotation érotique est manifeste, cette nudité festive est résolument exhibitionniste. Pour les communautés gay, lesbienne, écologiste ou naturiste ces parades offrent des conditions idéales de visibilité. Le corps se fait alors signe et message, le nu sert d'accroche, un peu à la manière d'une publicité, il suscite un choc et appartient à la démesure festive, dans ce contexte exceptionnel, la loi est permissive.

L'exhibition de la nudité porte aussi d'autres messages plus politiques et idéologiques. Apparus au détour des années 90 , on peut dire que la nudité appartient aujourd'hui au dispositif du discours contestataire. A Göteborg en Suède en 2000, à Québec en 2001, A Santiago du Chili en 2002, Sydney la même année, plus récemment en France en Mai 2007, chaque année partout dans le monde s'organise ce type de manifestions autour de revendications qui sont le fait de militants anti-mondialisation, opposants à la guerre (Irak par exemple), adeptes des pistes cyclables en France et en Espagne...La puissance d'évocation de la nudité produit un discours d'un nouveau type tout aussi mondialisé que ce qu'il dénonce. Le corps nu comme lieu de parole (inscriptions sur le corps de lettres) comme espace de représentation dans la sphère publique devient métaphore et allégorie. 


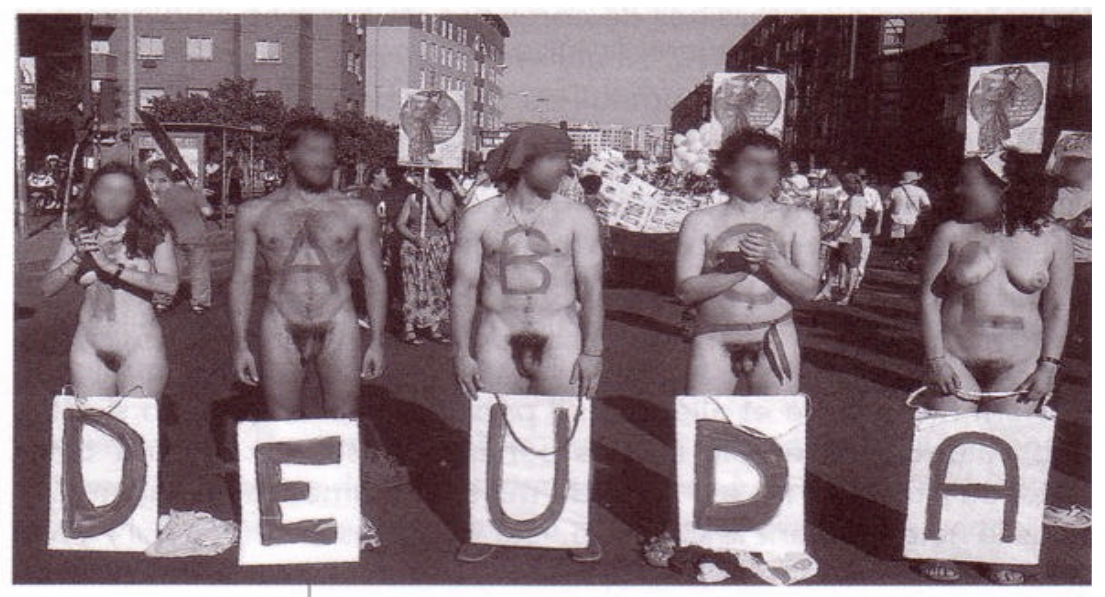

Il personnifie (il incarne au sens premier du terme) une idée, un slogan, une volonté. Ainsi les militants écologistes de la planète terre utilisent cette forme de manifestation pour affirmer le slogan suivant: «streakers for the wilderness», nature contre culture, nudité contre uniforme, la confrontation est aussi d'ordre symbolique.

Ainsi, la nudité affichée dans les lieux publics apparaît comme un phénomène complexe, animé par des forces contradictoires et fluctuantes, étroitement dépendante d'une contexte culturel personnel et collectif. Le nu exposé en public mobilise des facteurs psychologiques profonds, dans la société occidentale par exemple, le nu originel a été habillé, le retrouver à l'occasion n'est donc pas anodin. S'il s'agit du bronzage c'est le regard sur soi et celui des autres qui confère au nu une fonction spéculaire. S'il est lié à une préférence sexuelle, il vaut comme élément de séduction et comme affirmation d'une identité. S'il est mis en scène comme une transgression il développe des valeurs exhibitionnistes et ludiques et retrouve ainsi sa fonction carnavalesque d'autrefois. il peut aussi incarner un message politique, en misant sur l'appétit de spectaculaire et devenir l'expression d'un engagement, d'un refus radical. Le nu capte et transforme l'usage d'un espace public à son seul profit, occultant au passage ses normes et sa structuration habituelles, ses références culturelles et sociologiques. De ce point de vue, la nudité affichée équivaut à un détournement qui transforme l'espace public en scène. Le regard devient alors unité de mesure, il hiérarchise et donne un sens à ce qui est représenté. Ce dernier constat souligne bien le caractère aléatoire de l'espace public et la fragilité de son statut qui peut s'altérer ou disparaître devant le plus simple appareil.

\section{Le nu et la construction des genres}

La nudité sert de mode d'identification pour définir, à partir d'un modèle de référence : I'Autre, et lui attribuer par la même occasion des fonctions, des qualités, des rôles sociaux. A la naissance I'individu est situé dans l'une ou l'autre des classes sexuelles. Et c'est le point de départ d'une opposition mâle/femelle dont s'empare la société, elle se transforme en division sociale. C'est donc à partir d'une donnée biologique affirmée par le sexe féminin ou masculin que le corps nu a été surchargé 
Géographie et nudité: un mariage extravagant? (quelques

pistes de refléxion sur la production sociale de l'espace), pp. 152 - 169

d'enveloppes (les vêtements entre autre), de conventions, de prescriptions qui ont abouti à la notion de genre. Le genre est donc une construction sociale qui renvoie à une culture et se réfère à une classification d'un autre ordre que la classification biologique. Chaque société semble se faire sa propre conception de ce qui est essentiel et caractéristiques de ces deux classes sexuelles. Cette conception comporte des attributs appréciés et d'autres dépréciés. Il existe des idéaux de masculinité et de féminité, des interprétations de la nature humaine qui procurent des moyens d'identification. Être féminine ou viril n'a donc rien de naturel et automatique et ne dépend pas d'une quelconque composition de chromosomes, cela se travaille, se mérite et se juge.

La représentation de la nudité a joué un rôle déterminant dans les processus de constructions sociales des genres en imposant des stéréotypes de corps qui incarnent des valeurs plus que des réalités. Virilité comme maternité (ou féminité) ne renvoie pas au corps réel de I'homme ou de la femme. La nudité esthétisée par le travail du plasticien ou du publicitaire arrache le corps à la réalité. La figure de ces nus devient dès lors un corps idéal en même temps qu'on idéal à atteindre. C'est donc sous la forme d'images de nudités que féminité et masculinité se donnent à lire.

Le nu artistique avec tout son arsenal de normes académiques apparaît comme le support idéal, le porte-enseigne de processus de constructions sociales et idéologiques dans les sociétés occidentales qui fonctionnent, à partir de la renaissance sur le mode du regard et de la représentation. Chaque époque propose une version idéalisée du corps qui informe sur le rôle attribué à chaque sexe. L'exploration des figures de nu féminin est assez éloquente à cet égard : Que de changements depuis la préhistoire, à
I'Aurignacien les Vénus étaient à proprement parler monstrueuses, fesses, seins, vulve envahissent le corps, de là à conclure que les femmes de la préhistoire étaient toutes obèses, il n'y a qu'un pas... qu'il convient de ne pas franchir ! l'argument du nu était juste là pour appuyer la sacralisation de la féminité en participant sans doute d'un rite de fécondité ${ }^{1}$. Au moyen âge, puis plus tard à la Renaissance les grands maîtres schématisent les attributs féminins dans la représentation d'Êve ou de Vénus (Botticelli, la Naissance du printemps). Les seins sont menus, les courbes du corps sont molles, une nouvelle femme idéale voit le jour et elle n'est plus seulement la mère du genre humain. Les schématisations des images de nus se succèdent dans tout l'occident chrétien et tendent à se rapprocher de la mode et de la parure. L'une et l'autre s'influencent réciproquement. Aujourd'hui encore par l'intermédiaire des photos de nus publicitaires affichées dans la presse magazine, l'idéal féminin se définit à travers les (non) formes d'une femme maigre (mince), à la silhouette longiligne, aux jambes interminables et à la poitrine pigeonnante. Elle s'incarne parfaitement dans une figure, celle de la poupée Barbie que les petites filles habillent ou déshabillent à leur guise.

Du côté masculin, l'idéal de la beauté peine à se définir. Il faut attendre la fin du XVIIIe siècle pour assister à l'émergence de l'idée même de beauté. Cet idéal est fondé sur un assemblage de canons esthétiques qui varient peu et font référence à la Grèce antique. L'Allemand Winckelmann fut le propagateur de ce modèle idéal, I'Apollon du belvédère comme l'Hermès de Praxitèle devinrent les modèles d'un nouveau culte de la virilité. Ces sculptures, parce qu'elles montraient des nus étaient vues comme des abstractions de la beauté. Une valorisation de la beauté masculine inédite dans une culture ou la beauté ne pouvait être que l'apanage des nus féminins. 
La nudité dans l'histoire de l'art, déclinée sous toutes ses formes a chaque fois servi à définir des canons de la beauté associées souvent à des valeurs morales. A chaque modèle masculin ou féminin s'applique un certain nombre de valeurs, elles prennent souvent la forme d'allégories ou de symboles. Les nus féminins incarnent la sensualité, l'érotisme. Les nus masculins signalent la force, le courage, la puissance, I'héroïsme, emblèmes de la virilité...et ces valeurs se lisent sur les corps: aux musculatures puissantes font écho les courbes lisses et les poitrines généreuses. De ce point de vue, les figures masculines comme féminines furent chargées de symboles pour représenter la Nation. En général les hommes incarnaient les valeurs normatives: le travail, la guerre, le courage, les femmes les qualités maternelles qui renvoyaient à la tradition, elles devaient rappeler une certaine stabilité, d'ou leur aspect souvent monumental (Germania, Marianne, Brittania).

Les régimes totalitaires de l'entredeux guerres illustrent cette formidable capacité de la nudité artistique à incarner des modèles de l'Homme Nouveau (nu). En revanche, la représentation de la femme (habillée) est restée conforme à un modèle classique de mère. Le fascisme, puis le Nazisme on réinvestit les valeurs associées à la virilité et mettant en avant les vertus de la discipline, de l'action et de la camaraderie élevées au rang suprême de valeurs morales. Le nu, reflet de la beauté classique grecque, devint le symbole du national-socialisme, de l'homme nouveau. Dans L'Italie de Mussolini comme dans I'Allemagne d'Hitler le nu masculin devint un symbole politique, un idéal.

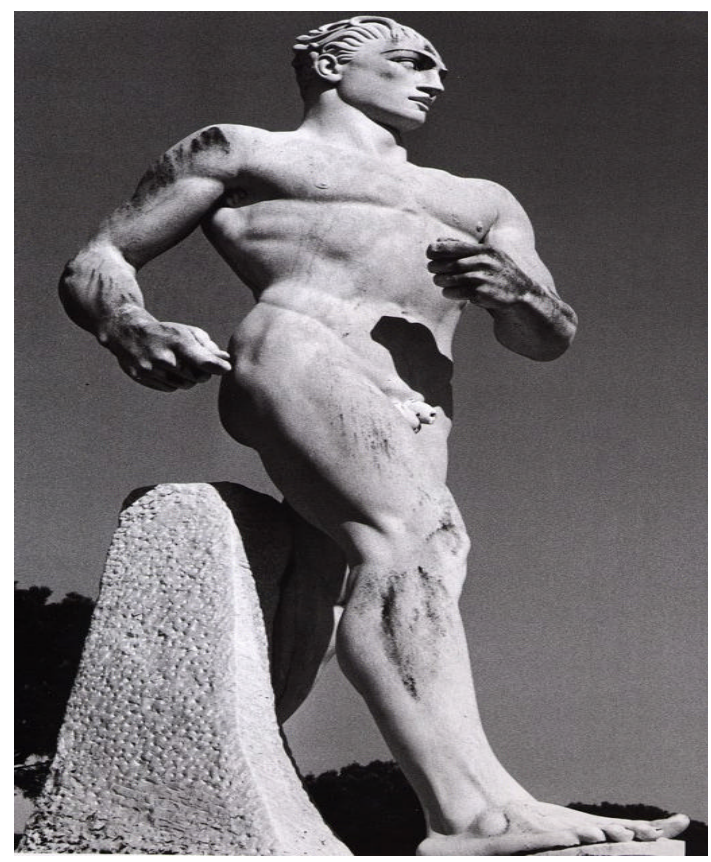

Cette nudité devait se conformer au modèle grec et cela exigeait des soins attentifs car le nu rappelait l'existence d'un monde sain, antérieur à la modernité. Le développement de ces stéréotypes a 
Géographie et nudité: un mariage extravagant? (quelques

pistes de refléxion sur la production sociale de l'espace), pp. 152 - 169

accompagné celui du racisme et de l'antisémitisme. Juifs, gitans, criminels, fous, homosexuels...tous décrits comme également laids, disproportionnés, agités et honteux de dévoiler leur nudité, tous représentés de manière grotesque à ces persécutés on imposa violemment la nudité comme forme de torture, d'humiliation et de déshumanisation dans les camps. Tous ces contretypes, considérés par le régime nazi comme des sous-hommes seront marqués dans leur chair par des numéros et beaucoup finiront dans les chambres à gaz intégralement nus.

Si on s'est servi du corps masculin comme emblème pour montrer ce que devait être un homme nouveau, il n'a pas été possible d'en faire autant pour les femmes qui sont restées assignées à leur rôle de mère, d'épouse ou de gardienne du foyer. L'idéal de la beauté féminine a suivi d'autres voies. Avec l'arrivée des processus de reproduction et la photographie, la représentation de la nudité féminine se doubla de deux autres types de modèles celui de la prostituée et celui de la femme fatale qu'on retrouvait dans les revues de charme ou les revues pornographiques mais on vit aussi émerger un nouveau type de femmes: la femme «sauvage » dans une presse tout à fait convenable.

Dans un article intitulé « voyeurs de lim périalism e » Tam ar $Y$ Rothem berg s'est intéressé aux représentations des images féminines des peuples sauvages à travers le très sérieux National geographic entre 1889 et 1954 . Véritable institution, cette revue vendue à plusieurs millions d'exemplaires donnait à voir à l'Amérique blanche et puritaine les corps dénudés des hommes et des femmes de cultures primitives. Sous des dehors très ethnographiques, le national geographic donnait à voir des nudités dans l'enceinte privée de la maison (et non pas exposé dans des musées) ou dans les salles d'attente des médecins. Les corps féminins, africains; asiatiques, amazoniens étaient considérés comme plus proche de la nature en vertu des cultures qu'ils représentaient ou de leur couleur de peau. "C'était la race qui distinguait les femmes pouvant être photographiées nues des autres. Les femmes de couleur étaient bien des femmes.. mais elles appartenaient à un type différent de femmes ... elles différaient tellement de la femme américaine, implicitement blanche, de préférence protestante et appartenant à la classe moyenne qu'on pouvait tranquillement la placer dans une autre catégorie, inférieure ». Le contraste entre l'américaine, hautement civilisée, toute en retenue et cette femme sans pudeur, complètement sauvage, qui montrait ses seins était de nature à justifier auprès du national geographic comme de ses lecteurs la diffusion de ces photos de femmes «autres». Le racisme appartient à la convention narrative dont le national geographic a été un des vecteurs au cours du XXe siècle en alimentant le fantasme de la femme sauvage. La société américaine, blanche et civilisée en exhibant dans les pages glacées de ce magazine ces femmes sauvages, sous des dehors de curiosité très convenable trouvait là le moyen d'un voyeurisme à bon marché.

L'étude de la nudité traversé par la question du genre pose avec acuité le problème de la différence et celui de I'inégalité, car être différent n'implique pas nécessairement être subordonné. Si les corps sexués, de couleur, de civilisation autre montrent bien des différences, il ne s'agit en aucun cas de valeurs. Le nu n'enseigne rien, mais tous les discours sur sa valeur sont l'indice d'une tentative de classement, de mise en ordre, de hiérarchie. 


\section{Quand le nu habille les murs: déconstruction du discours publicitaire}

Le spectacle de la nudité publicitaire affichée sur les murs appartient aujourd'hui aux paysages urbains et à la vie quotidienne des citoyens d'une grande partie du monde. La présence ou l'absence de ces nus rend compte de clivages culturels, il en est du même coup une forme de témoignage. Son traitement et sa fréquence sont en étroite corrélation avec les grandes aires culturelles et religieuses. La globalisation à l'œuvre sur la planète ne fonctionne pas sur ce sujet, les représentations d'images de nu définissent une frontière au delà de laquelle les sensibilités culturelles se manifestent de manière affirmée. Le monde islamique, I'Afrique et une grande partie de I'Asie n'utilisent pas la nudité comme argument pour cause de tabous. Il n'est pas question dans cette partie de l'exposé de faire l'apologie de la publicité mais plutôt de rendre compte de son impact sur la vie quotidienne. En effet, la publicité se déploie dans tous les espaces, publics ou privés, elle est ubiquiste et en même temps touche tous les publics. Le cinéma, et les arts plastiques en général ont leurs lieux réservés, parfois confidentiels, tout comme ils ont leur public. Il n'en va pas de même pour le nu publicitaire: télévision, murs des villes, presse consultée dans les salles d'attente que tout un chacun fréquente dans sa vie quotidienne sont autant de lieux où il est possible et même inévitable de voir des nus. Figure omniprésente du paysage urbain, il contribue à la définir et en fixe les horizons.

Grâce aux techniques de reproductions, le nu publicitaire est partout et nulle part, les campagnes d'affichages produisent des séries d'images qui se donnent à voir à un moment ici et ailleurs, sur le bord des routes, dans les villes, en grand et petit format, la série produit une norme et un conformisme qui contribuent à ce que toutes les villes se ressemblent, le nu crée une ponctuation visuelle, un rythme dans le paysage. Ce que nous pouvons observer dans la rue, se retrouve dans les pages d'un magazine, à la télévision, il est omniprésent pendant quelques semaines, puis disparaît de notre espace de vie quotidien pour réapparaître ailleurs, sous une autre forme. Mais finalement c'est toujours la même image de nu qui nous est donnée à voir, reposant indéfiniment la question de l'altérité. ce corps de femme (ou d'homme) nu renvoie à l'image d'un corps générique féminin, il n'est le corps de personne. Il ne permet pas un processus d'identification, mais définit le corps de toutes les femmes.

Si l'affichage urbain rend le nu publicitaire omniprésent dans les espaces publics, le passage dans l'espace privé n'interrompt pas le phénomène. A peine rentré chez soi, il apparaît sous d'autres formes, sur d'autres supports. On pourrait supposer que l'assignation faite au regard est moins prégnante, qu'il y a davantage de liberté laissée au regard! En réalité, même si tout le monde n'achète pas le même journal, ne lit pas les mêmes magazines, ne regarde pas les mêmes chaînes de télévision aux mêmes heures, le nu s'immisce par le petit écran dans les espaces privés, se retrouve dans les salles d'attente des dentistes, des coiffeurs... Ce véritable continuum engage d'autres types de regards, d'autres postures des corps. A la différence du nu affiché dans la rue, qui interpelle n'importe quel public celui-ci est soigneusement référencé par rapport à une cible précise. En fonction du type de spectateur ou du lectorat de leur presse, les publicitaires proposent des nus coïncidant avec un public précis. Ainsi dans les journaux masculins, les images de femmes nues sont légion surtout dans des situations érotiques (le pendant existe dans la version presse spécialisée pour les Gays avec des hommes nus). Dans les journaux féminins, les produits de beauté et autres articles supposés séduire les femmes sont proposés par des modèles aux plastiques parfaites 
Géographie et nudité: un mariage extravagant? (quelques

pistes de refléxion sur la production sociale de l'espace), pp. 152 - 169

mais dont l'attitude au moins sexuellement n'est pas équivoque. Le même procédé est à l'œuvre dans les revues spécialisées en puériculture, ou l'on trouve le plus de nus de nourrissons attendrissants. Un protocole identique se déroule pour la publicité à la télévision où les nus sont différents suivant les heures d'écoute.

Le nu est un attrape regard dont la prolixité, la richesse et la complexité des valeurs assurent au discours publicitaire la certitude de capter l'attention, quelque soit la nature du produit proposé. La publicité est cannibale, elle se nourrit de la société. En même temps elle est caméléon et s'imprègne des valeurs du moment. C'est d'ailleurs ce qui rend vraisemblable le discours publicitaire, il refabrique un monde artificiel, mais conserve un effet de réalité. La révolution des moeurs, comme un certain affranchissement des normes liées à la pudeur attestent de cette imprégnation restitution du discours publicitaire tout en l'accentuant. les images de nus classiques fortement emprunts du codage artistique, même s'ils ne sont pas abandonnés, cèdent aujourd'hui la place à des représentations de corps nus plus « naturels», en extérieur, ou en action. Même si l'image cliché de la jeune femme nue est encore largement employée, elle laisse la place à d'autres figures de nus. Quant au nu masculin, jusqu'alors réservé à quelques produits généralement liés à l'hygiène ou au parfum, il s'impose depuis peu sur tous les supports médiatiques. Cette présence rend compte, pour une part de l'émergence du mouvement homosexuel masculin qui revendique une visibilité et sort ainsi du ghetto. Cette nouvelle «cible » qui possède une presse spécialisée, se montre désormais au grand jour. Le fort pouvoir d'achat et les habitudes de consommation de cette clientèle font l'objet de soins particulièrement attentifs de la part des annonceurs. C'est sans doute une des explications de la mutation profonde des représentations de la nudité masculine depuis quelques années.
En utilisant le nu, les publicitaires mobilisent un champ de valeurs extrêmement vaste où se mêlent esthétique, sexualité, fantasme, identité, normes et messages mercantiles. A travers leur discours, verbal ou iconographique, ils déclenchent des associations, des comportements qui remettent en cause les repères établis, repères entre espace public et espace privé, entre intimité et extimité entre soi et les autres. Il serait cependant excessif de croire que le souffle publicitaire balaye tout sur son passage. En définitive si par le biais du nu, la publicité puise inlassablement dans les travers de la société, si elle fouille dans ses replis pour mieux nous émouvoir, nous choquer, ou nous faire rire, elle donne d'abord à lire notre rapport au corps et au nu, à l'intimité et à la sexualité, au réel et à l'imaginaire. Elle propose un raccourci commode de nos fantasmes et de nos désirs à travers une lecture de la nudité.

Conclusion: La nudité pour être comprise ne peut s'appréhender que par rapport à un environnement, elle demande à être située, à être quelque part. Une fois définie comme un état du corps, elle ne peut ensuite s'interpréter que par rapport à un espace dans lequel elle se donne à lire. Mais à l'inverse, elle donne aussi du sens aux lieux dans lesquels elle se manifeste. Ce système d'influences réciproques est au cœur d'un processus d'organisation de l'espace. Ici et ailleurs, aujourd'hui comme hier, la nudité habite les lieux qu'elle produit comme elle s'impose et fait de la contrebande ailleurs là où on ne l'attend pas. Cependant l'objet-nudité demeure assez insaisissable, même s'il apparaît entouré de normes qu'on croit immuables, comme celles liées à l'hygiène ou à la toilette. Le corps nu se replie dans l'intimité de quelques pièces de la maison marquant ainsi ce qui est privé de ce qui ne l'est pas, comme il peut surgir au détour d'une rue dans les tumultes d'une parade. Avec le nu, c'est le regard qui est mis en jeu. Un regard porteur des attributs d'une civilisation, éduqué par des normes 
sociales, par un système de conventions; un regard qui hiérarchise, qui s'interroge, qui s'émoustille ou qui sourit. Pour se saisir de la nudité comme objet géographique peutêtre faut-il tout simplement savoir où on se trouve et d'où on regarde?

\section{Notas}

1 Géographie de la nudité : être nu quelque part, Francine Barthe-Deloizy, éditions Bréal 2004, 239 pages

2 «Ils sont nus comme leur mère les enfanta », Journal de Christophe Colomb, La découverte de I'Amérique, 4 volumes, Paris la découverte, 1979 réédition. On trouve aussi de nombreux passages dans Le voyage autour du Monde de L.A De Bougainville, Paris Collection Folio, 1982

${ }^{3}$ M.A Descamps, Le nu et le vêtement, Paris, 1972, Editions universitaires.

${ }^{4}$ Les zoos humains Baratay et Hardoin Fugier, Paris La découverte, 1998

${ }^{5}$ Le nu et le vêtement, M.A Descamps, 1972, éditions universitaires

${ }^{6}$ Encyclopédie de l'amour en Islam, Malek Chebel, tome 1 et 2 Petite bibliothèque Payot, Paris 1995

${ }^{7}$ La dimension cachée, E.T Hall, Seuil, 1971
8 Nudité et pudeur, le mythe du processus de civilisation, Hans Peter Duerr, Paris MSH, 1998

${ }^{9}$ Nous empruntons ici ce vocable dans le sens ou le définit Jacques Lévy dans Logiques de l'espace, esprits des lieux, Belin, Paris 2000

10 Histoire du naturisme, Arnaud Baubérot, 2006, Presses universitaires de Rennes

11 «Géographie du naturisme, la recherche de I'Eden » dans la revue Géographie et cultures N 37, Paris I'harmattan, 2000 et « Le naturisme des cures atmosphériques au tourisme durable », dans Communications

${ }^{12}$ Alain Roger, Nus et paysages, essai sur la fonction de l'art, Paris, Aubier, 1978

13 « voyeurs de l'impérialisme : le magazine National geographic avant la deuxième guerre mondiale » dans Godlewska A et Smith N (sous la direction) : Geography and empire: critical Studies in the history of geography, Oxford, Blackwell, 1993

\section{Bibliografia}

Authier C, Le nouvel ordre sexuel, Paris Bartillat, 1998

Baratay E, Hardoin-Fugier E, Les zoos, histoire des jardins zoologiques en occident (XVIe-XXe siècle), Paris, La découverte, 1998

Barthe-Deloizy F, Géographie de la nudité : être nu quelque part, Paris, Bréal, 2003

Barthe-Deloizy F, Géographie du naturisme, la recherche de I'Eden, dans Géographie et cultures No 37, Paris, L'Harmattan, 2000

Barthe-Deloizy F, Le naturisme des cures atmosphériques au tourisme durable, dans Communications, N 74, Paris, Seuil, 2003
Barthes R, Système de la mode, Paris, Seuil, 1967

Barthes R, Mythologies, Paris, Seuil, 1957

Baubérot A, Histoire du naturisme, Rennes, Presses universitaires de Rennes, 2005

Bologne JC, Histoire de la pudeur, Paris, Olivier Orban, 1986

Bourdieu P, La domination masculine, Paris, Seuil, 1998

Brohm JM, Corps et politique, JP Delage, 1978 Chebel M, L'encyclopédie de I'amour en Islam, T1 et 2 Paris, Payot, 1995 
Géographie et nudité: un mariage extravagant? (quelques

Claval P, Géographie culturelle, Paris Armand Colin, 2003

Claval Paul, Le smythes fondateurs des sciences sociales, Paris PUF, 1990

Colomb C, La découverte de I'Amérique, 4 vol, Paris, La découverte, 1979

Descamps MA, Le nu et le vêtement, Paris, Editions universitaires, 1972

Descamps MA, Psychosociologie du naturisme, Paris, Trimégiste, 1982

Detrez C, La construction sociale du corps, Paris, Seuil, 2002

Duerr HP, Nudité et pudeur, le mythe du processus de civilisation, Paris, MSH, 1998

Elias N, La civilisation de smoeurs, Paris, Calman,-Lévy, 1973

Goffman E, La mise en scène de la vie quotidienne, 2 vol Paris, éditions de minuit, 1973

Goffman E, L'arrangement des sexes, Paris, éditions la Dispute, 2002

Hall ET, La dimension cachée, Paris, Seuil, 1971 Héritier F, Masculin/féminin, la pensée de la différence, Paris Odile Jacob, 1996
Kaufmann JC, Corps de femmes, regards d 'hommes, sociologie des seins nus, Paris, Presses de la cité, 1998

Lévy J, Le tournant géographique, Penser l'espace pour lire le monde, Paris, Belin, 1999

Lévy J, Lussault M, Logiques de l'espace, esprits des lieux, Paris, Belin, 2000

Mauss M, Sociologie et anthropologie, Paris, PUF, 1950

Merleau-Ponty $M$, Phénoménologie de la perception, chapitre 5 le corps sexué, Paris Gallimard, 1945

Roger A, Nus et paysages, essai sur la fonction de I'art, Paris, Aubier, 1978

Rothemberg TY, Voyeurs de I'impérialisme, le magazine National geographic avant la deuxième guerre mondiale, dans Geography and empire : critical studies in the history of geography, Oxford, Blackwell, 1993

Viard J, Court traité des vacances, les voyages et I'hospitalité des lieux, Paris, édition de I'Aube, 2000

Vigarello G, Le propre et le sale, I'hygiène du corps depuis le moyen âge, Paris, Seuil 1985 